\title{
Adaptive Proportional Integral Observer Design for Interval Type 2 Takagi-Sugeno Fuzzy Systems
}

\author{
Atef Khedher $\mathbb{D}^{\mathbb{D}}{ }^{1}$ Ilyes Elleuch, ${ }^{2}$ and Kamal BenOthman ${ }^{3}$ \\ ${ }^{1}$ Lara Automatique, ENIT, Tunis, Tunisia \\ ${ }^{2}$ University of Sousse, Sousse, Tunisia \\ ${ }^{3}$ LASEE, ENIM, Monastir, Tunisia \\ Correspondence should be addressed to Atef Khedher; khedher_atef@yahoo.fr
}

Received 17 May 2021; Revised 28 June 2021; Accepted 2 July 2021; Published 16 July 2021

Academic Editor: Omar Naifar

Copyright $\odot 2021$ Atef Khedher et al. This is an open access article distributed under the Creative Commons Attribution License, which permits unrestricted use, distribution, and reproduction in any medium, provided the original work is properly cited.

In this paper, the problem of fault estimation in systems described by Takagi-Sugeno fuzzy systems is studied. A proportional integral observer is conceived in order to reconstruct state and faults which can affect the studied system. Proportional integral observer can easily estimate actuator faults which are assimilated to be as unknown inputs. In order to estimate actuator and sensor faults, a mathematical transformation is used to conceive an augmented system, in which the initial sensor fault appears as an unknown input. Considering the augmented state, it is possible to conceive an adaptive observer which is able to estimate the whole state and faults. The noise effect on the state and fault estimation is also minimized in this study, which provides some robustness properties to the proposed observer. The proportional integral observer is conceived for nonlinear systems described by Takagi-Sugeno fuzzy models.

\section{Introduction}

State estimation can have numerous applications in control and diagnosis. In often cases, the system state is globally or partially unknown, so its estimation can be a solution.

Generally, the process is affected by disturbances, measurement uncertainties, and sensor and/or actuator faults. Disturbances and faults are usually considered as unknown inputs which can have a random behavior in time, and they can have harmful effects on the process. Observers with unknown inputs were the subject of many works [1-3]. Indeed, methods of simultaneous estimation of the unknown inputs and the system state were proposed in [3]. In [1], authors present a method of simultaneous estimations of system state and unknown inputs and outputs, and it is considered that some outputs are not accessible to measure. In [2], a comparison study is proposed between sliding mode observers and unknown inputs' observers in the context of fault estimation, but only the actuator fault is considered.

Takagi-Sugeno fuzzy systems, named also multiple models [4], are an efficient approach to handle complex nonlinear systems $[5,6]$. They are composed of a set of linear models weighted by nonlinear activation functions verifying the convex-sum property $[6,7]$. Using the same activation functions, nonlinear observers can be designed to make the state estimation. These observers are called multiple observers $[5,8]$. Indeed, works presented in $[6,7]$ can be considered as the first works regarding this kind of models. It is proved in these works that these models can approximate well the nonlinear system behavior. Works presented in $[5,8]$ are interested in some application of state and fault estimation using this kind of models.

Approaches using Takagi-Sugeno fuzzy models are the subject of numerous works [9-12], dealing with state estimation in the presence of unknown inputs or parameter uncertainties. In [9], authors propose to consider singulary perturbed Takagi-Sugeno models where the activation function is depended on unmeasurable variables such as the system state. In [10], a case of the switching system is considered as a particular form of Takagi-Sugeno models. In this kind of models, the activation function can be 0 or 1 . In [11], authors are interested in Takagi-Sugeno-Kang models 
for online identification with application to crane systems. Sensor networks are considered in [12] more precisely in the case of nonfragile distributed filters with Takagi-Sugeno models. In this context, the problem of state and fault estimation is studied in $[2,4,5,8,13]$. In [4], a sliding mode observer with unknown input for the case of uncertain Takagi-Sugeno models is proposed. In [5], a method of state and unknown input estimation is presented for multiple models. In [8], a method of sensor faults' estimation is proposed for systems described by Takagi-Sugeno models. For linear systems, Edwards proposes, in [2], to use a mathematical transformation in order to conceive a new system where the sensor fault appears as an unknown input. This transformation is used next in [14] for the fault estimation in the context of linear systems.

It is possible to estimate simultaneously the system state and the fault affecting the system using the proportional integral observer. This kind of observer is composed of two estimators (proportional and integral) $[4,5,8,13]$. In practice, the design of the proportional integral observer is reduced to the computation of the two gains (proportional and integral) where the proportional term lets to estimate the system state and the integral term permits to estimate the fault $[4,5,8,13]$. Works presented in $[4,5,8,13]$ are interested in state and fault estimation in the context of Takagi-Sugeno systems using proportional integral observers which are composed of two estimators; the first one called proportional terms is used to estimate the system state and the second one called integral term allows estimating the fault affecting the system. Some academic and real applications are given like the application to the model of turbo-reactor presented in [4].

Takagi-Sugeno fuzzy models can be of type 1 or 2 [15-18]. A type 2 fuzzy set uses upper and lower primary membership functions and a secondary membership function [15-18]. Contrary to a type 1 fuzzy set which has only one primary membership function, by consequent, a type 2 fuzzy set is more able to handle uncertainties and ambiguities. Type 2 fuzzy sets were proposed by Zadeh in 1975, but there were not many researchers interested in them until these last years. Some researchers started to consider type 2 fuzzy systems in the past several years due to their relative novelty [15-18]. Works presented in [15-18] are interested in type 2 Takagi-Sugeno fuzzy models. Indeed, it is shown that this type can be used to reduce the system complexity and the number of local models comparing with type 1 . The main difference between type 1 and type 2 is in the form of activation functions. Indeed, activation functions for type 1 are characterized by a real term for each time. For type 2 , in each time, the activation functions are characterized by fuzzy sets which are defined often in cases by their upper and lower bounds which is why they are called type 2 interval Takagi-Sugeno fuzzy systems. For each time, activation function is varying between upper and lower bounds.

Many other works focus on the state and fault estimation in several contexts. Let us cite briefly some of them and give the difference between them and the present work. In $[19,20]$, authors are interested on multiagent systems which are modeled by several agents where each agent presents a nonlinearity which is different from the principle of the
Takagi-Sugeno models where the local models are linear and the nonlinearity is given by the activation functions. The obtained results in these works are important but the main difference is in the used model. Multiagent systems are also considered for fault estimation in [21] by considering distributed $\ell_{1}$ state and fault estimation. Asymptotic fault and state estimation is proposed in [22] in the context of nonlinear systems which is different from this work where systems are modeled by Takagi-Sugeno models. The same work is extended to conceive fault tolerant control. In [23], Lipschitz condition is assumed for the state and fault estimation in the context of Takagi-Sugeno models, and these conditions are not considered in this work. In [24], only actuator fault is estimated simultaneously with the state estimate in the context of interval Takagi-Sugeno systems contrary with this work where both actuators and sensors' faults are considered. The Takagi-Sugeno discrete model is considered in [25-27], but in this work, we focus on continuous models. In [28], Takagi-Sugeno models with delay assumptions are considered; in this work, we do not consider delay. The application of fault estimation to the fault tolerant control is given in $[29,30]$. Ellipsoidal bounding conditions are assumed in [31].

The main contribution in this work is to extend the method of simultaneous estimation of the system state and actuator and sensor faults developed in the context of type 1 Takagi-Sugeno fussy systems for the case of type 2 Takagi-Sugeno fuzzy models. Indeed, the structure of type 2 Takagi-Sugeno fuzzy systems based on varying activation functions lets the extension of obtained results in the case of type 1 Takagi-Sugeno systems not evident since type 2 models are based on double fuzzy sets which are the model and the activation functions, which make the presented work more important. An adaptive proportional integral observer is proposed and used to assure this estimation. Classically, this observer is used to estimate system state and unknown inputs. This paper shows that it is possible to adapt this observer to estimate, simultaneously, the system state, the actuator, and the sensor faults. Indeed, the mathematical transformation proposed in [2], for the case of linear systems and extended to the case of systems described by Takagi-Sugeno models in $[4,8,13]$, is adapted to interval type 2 Takagi-Sugeno models in this paper. Based on the adapted form of this transformation, an augmented system state is obtained. This augmented system presents a generalized unknown input which contains the initial sensor and actuator faults. At this level, a proportional integral observer able to estimate the augmented state and the generalized unknown input is proposed. The estimation of the initial sensor and actuator faults is reduced to the estimation of the generalized unknown input. This work presents the three possible cases of faults' estimation:

(i) State and actuator fault estimation

(ii) State and sensor fault estimation

(iii) Simultaneous estimation of state, actuator, and sensor faults

The paper is organized as follows. Section 2 recalls the principle of Takagi-Sugeno multiple models type 1 and type 2. Section 3 describes the design of the proportional integral 
observer for the state and actuator faults' estimation. This observer is adapted to sensor faults' estimation in Section 4. Section 5 proposes a method to estimate simultaneously system state, sensor, and actuator faults. An example of simulation, showing the quality of estimation, is given in Section 7.

\section{Takagi-Sugeno Fuzzy Systems}

2.1. Elementary Background on Type 1 Takagi-Sugeno Fuzzy Systems. Takagi-Sugeno fuzzy systems are an appropriate tool which permits to model large class of complex and nonlinear systems with a mathematical model which can be used for analysis [32, 33], control [34, 35], and observer design $[1,8,13,36]$. This approach is based on a decomposition of the system operating space into a finite number of operating zones. Hence, a simple linear model describes the system dynamic behavior inside each operating zone. The contribution of each submodel in the global model is quantified using a nonlinear weighting function which can have various structures. The submodels are associated in the state equation using a common state vector. This model has been proposed, in a fuzzy modeling framework, by Takagi and Sugeno [7].

Takagi-Sugeno fuzzy systems are based on the assumption that each nonlinear dynamic system can be simply described as the fuzzy fusion of many linear models, where each linear model represents the local system behavior around an operating point. A Takagi-Sugeno model is described by fuzzy IF-THEN rules which represent local linear inputs/outputs' relations of the nonlinear system. It has a rule base of $M$ rules, each having $p$ antecedents, where the $i^{\text {th }}$ rule is expressed as follows:

$$
R^{i}: \operatorname{IF} \xi_{1} \text { is } F_{1}^{i} \text { and } \ldots \text { and } \xi_{p} \text { is } F_{p}^{i},
$$

where $i \in\{1, \ldots, M\}, F_{j}^{i}(j \in\{1, \ldots, p\})$ are fuzzy sets and $\xi=\left[\xi_{1}, \xi_{2}, \ldots, \xi_{p}\right]$ is a known vector of premise variables [5] which may depend on the state, the input, or the output. Variable $\xi$ is called the decision variable.

The global Takagi-Sugeno fuzzy model is given by the aggregation of the submodels using the weighting functions as follows:

$$
\left\{\begin{array}{l}
\dot{x}(t)=\sum_{i=1}^{M} \mu_{i}(\xi(t))\left(A_{i} x(t)+B_{i} u(t)\right), \\
y(t)=\sum_{i=1}^{M} \mu_{i}(\xi(t)) C_{i} x(t),
\end{array}\right.
$$

where $x(t) \in \mathbb{R}^{n}$ is the state vector, $u(t) \in \mathbb{R}^{r}$ is the control vector, $y(t) \in \mathbb{R}^{m}$ is the vector of measures, and $A_{i}, B_{i}$, and $C_{i}$ are known constant matrices with appropriate dimensions.

The weighting functions $\mu_{i}(\xi(t))$ assure a progressive passage between the local models and verifies the property of the convex sum: $\sum_{i=1}^{M} \mu_{i}(\xi(t))=1, \forall t \quad$ and $0 \leq \mu_{i}(\xi(t)) \leq 1, \forall i=1, \ldots, M, \forall t$.

If, in the equation of the output, it is supposed that $C_{1}=C_{2}=\cdots=C_{M}=C$, the output of the multiple model
(2) is reduced to $y(t)=C x(t)$, and the multiple model state equation becomes

$$
\left\{\begin{array}{l}
\dot{x}(t)=\sum_{i=1}^{M} \mu_{i}(\xi(t))\left(A_{i} x(t)+B_{i} u(t)\right), \\
y(t)=C x(t) .
\end{array}\right.
$$

2.2. Type 2 Takagi-Sugeno Fuzzy Systems. Interval type 2 Takagi-Sugeno fuzzy models are nonlinear systems with $M$ rules, where the Rule $R^{i}$ is as follows.

IF $f_{1}(\xi(t))$ is $\widetilde{F}_{1}^{i}$ AND ...AND $f_{p}(\xi(t))$ is $\widetilde{F}_{p}^{i}$ THEN

$$
\left\{\begin{array}{l}
\dot{x}(t)=A_{i} x(t)+B_{i} u(t) \\
y=C_{i} x(t)
\end{array}\right.
$$

where $f_{j}(\xi(t))$ is the premise variable depending on a known decision variable $\xi$ and $\widetilde{F}_{i}^{j}$ is an interval type 2 fuzzy set, for $i \in\{1,2, \ldots, M\}$ and $j \in\{1,2, \ldots, p\}, p$ is a positive integer, $x(t) \in \mathbb{R}^{n}$ is the state vector, $u(t) \in \mathbb{R}^{q}$ in the system input, $y(t) \in \mathbb{R}^{l}$ is the system output, and $A_{i}, B_{i}$, and $C_{i}$ are known matrices with appropriate dimensions.

The $i^{\text {th }}$ fuzzy rule can be described by the interval sets: $\mu_{i}(\xi(t))=\left[\underline{\mu}_{i}(\xi(t)), \bar{\mu}_{i}(\xi(t))\right], i \in\{1,2, \ldots, M\}, \quad$ where $\underline{\mu}_{i}(\xi(t))=\prod_{j=1}^{M} \underline{\mu}_{F_{j}}\left(f_{i}(\xi(t))\right) \geq 0$ and $\bar{\mu}_{i}(\xi(t))=\prod_{i=1}^{M} \bar{\mu}_{F_{j}}$ $\left(f_{i}(\xi(t))\right) \geq 0$ are the lower and upper grades of membership, respectively. $\bar{\mu}_{\widetilde{F}_{j}}\left(f_{i}(\xi(t))\right) \geq 0$ and $\underline{\mu}_{F_{j}}\left(f_{i}(\xi(t))\right) \geq 0$ denote the lower and upper weighting functions, respectively. Therefore, it is assumed that $\bar{\mu}_{F_{j}}\left(f_{i}(\xi(t))\right) \geq \underline{\mu}_{F_{j}}\left(f_{i}(\xi(t))\right) \geq 0$ and $\underline{\mu}_{i}(\xi(t)) \geq \bar{\mu}_{i}(\xi(t))$ for all $i$.

The interval type 2 Takagi-Sugeno fuzzy system is given by the following state equations:

$$
\left\{\begin{array}{l}
\dot{x}(t)=\sum_{i=1}^{M} \mu_{i}(x(t))\left(A_{i} x(t)+B_{i} u(t)\right), \\
y(t)=\sum_{i=1}^{M} \mu_{i}(x(t)) C_{i} x(t),
\end{array}\right.
$$

with

$$
\begin{aligned}
\mu_{i}(\xi(t)) & =\frac{\omega_{i}(\xi(t))}{\sum_{i=1}^{M} \omega_{i}(\xi(t))}, \\
\sum_{i=1}^{M} \mu_{i}(\xi(t)) & =1
\end{aligned}
$$

where

$$
\omega_{i}(\xi(t))=\underline{\vartheta}_{i}(\xi(t)) \underline{\mu}_{i}(\xi(t))+\bar{\vartheta}_{i}(\xi(t)) \bar{\mu}_{i}(\xi(t)) \geq 0, \quad \forall i
$$

The nonlinear functions $\underline{\vartheta}_{i}(\xi(t))$ et $\bar{\vartheta}_{i}(\xi(t))$ must verify the following conditions: 


$$
\begin{array}{r}
0 \leq \bar{\vartheta}_{i}(\xi(t)) \leq 1, \\
0 \leq \underline{\vartheta}_{i}(\xi(t)) \leq 1, \\
\bar{\vartheta}_{i}(\xi(t))+\underline{\vartheta}_{i}(\xi(t))=1 .
\end{array}
$$

To summarize, it is possible to write an interval type 2 Takagi-Sugeno system in the following form:

$$
\left\{\begin{array}{l}
\dot{x}(t)=\sum_{i=1}^{M} \mu_{i}(x(t))\left(A_{i} x(t)+B_{i} u(t)\right), \\
y(t)=\sum_{i=1}^{M} \mu_{i}(x(t)) C_{i} x(t),
\end{array}\right.
$$

where $\quad \sum_{i=1}^{M} \mu_{i}(\xi(t))=1, \forall t \quad$ and $\quad 0 \leq \mu_{i}(\xi(t)) \leq 1$, $\forall t, \forall i=1, \ldots, M$ and

$$
\mu_{i}(\xi(t))=\frac{\bar{\mu}_{i}(\xi(t)) \bar{\vartheta}_{i}(\xi(t))+\underline{\mu}_{i}(\xi(t)) \underline{\vartheta}_{i}(\xi(t))}{\sum_{i=1}^{M}\left(\bar{\mu}_{i}(\xi(t)) \bar{\vartheta}_{i}(\xi(t))+\underline{\mu}_{i}(\xi(t)) \underline{\vartheta}_{i}(\xi(t))\right)} .
$$

\section{Actuator Faults' Estimation}

The objective of this part is to conceive a proportional integral observer able to estimate actuator faults affecting nonlinear systems represented by interval type 2 Takagi-Sugeno models.

Consider the following interval type 2 Takagi-Sugeno fuzzy system affected by an actuator fault and a measurement noise:

$$
\left\{\begin{array}{l}
\dot{x}(t)=\sum_{i=1}^{M} \mu_{i}(\xi(t))\left(A_{i} x(t)+B_{i} u(t)+E_{i} f_{a}(t)\right), \\
y(t)=C x(t)+D w(t),
\end{array}\right.
$$

where $x(t) \in \mathbb{R}^{n}$ is the system state, $y(t) \in \mathbb{R}^{m}$ is the measured output, $u(t) \in \mathbb{R}^{r}$ is the input, $f_{a}(t)$ is the actuator fault which is assumed to be bounded, and $w(t)$ is the measurement noise. $A_{i}, B_{i}$, and $C$ are known constant matrices with appropriate dimensions. $E_{i}$ and $D$ are, respectively, the fault and noise distribution matrices which are assumed to be known. The scalar $M$ represents the number of the local models. $\mu_{i}(\xi(t))$ are the activation functions verifying equation (12).

The structure of the proportional integral observer is chosen as follows:

$$
\begin{aligned}
\{\dot{\hat{x}}(t) & =\sum_{i=1}^{M} \mu_{i}(\xi(t))\left(A_{i} \hat{x}(t)+B_{i} u(t)+E_{i} \hat{f}_{a}(t)+K_{i} \tilde{y}(t)\right), \\
\dot{\hat{f}}_{a}(t) & =\sum_{i=1}^{M} \mu_{i}(\xi(t)) L_{i} \tilde{y}(t), \\
\hat{y}(t) & =C \hat{x}(t),
\end{aligned}
$$

where $\hat{x}(t) \in \mathbb{R}^{n}$ is the estimated system state, $\widehat{f}_{a}(t)$ represents the estimated fault, $\hat{y}(t) \in \mathbb{R}^{m}$ is the estimated output, $\tilde{y}(t)=y(t)-\hat{y}(t), K_{i}$ are the local proportional observer gains, and $L_{i}$ are the local integral gains to be computed.

Let us define the state estimation error $\tilde{x}(t)$ and the fault estimation error $\tilde{f}_{a}(t)$. They are given by the following equalities:

$$
\begin{gathered}
\tilde{x}(t)=x(t)-\hat{x}(t), \\
\tilde{f}_{a}(t)=f_{a}(t)-\widehat{f}_{a}(t) .
\end{gathered}
$$

The dynamics of the state estimation error is given by the computation of $\dot{\tilde{x}}(t)$ which is written as follows:

$$
\dot{\tilde{x}}(t)=\dot{x}(t)-\dot{\hat{x}}(t)=\sum_{i=1}^{M} \mu_{i}(\xi(t))\left(A_{i}-K_{i} C \widetilde{x}(t)+E_{i} \tilde{f}_{a}(t)+K_{i} D w(t)\right)
$$

The dynamics of the fault estimation error is given by the expression of $\widetilde{f_{a}}(t)$ written below:

$$
\dot{\dot{f_{a}}}(t)=\dot{f}_{a}(t)-\dot{\hat{f}}_{a}(t)=\dot{f}_{a}(t)-\sum_{i=1}^{M} \mu_{i}(\xi(t))\left(L_{i} C \tilde{x}(t)-L_{i} D w(t)\right) \text {. }
$$

The following matrices are introduced: 


$$
\begin{aligned}
\varphi_{a} & =\left[\begin{array}{c}
\tilde{x}(t) \\
\tilde{f}_{a}(t)
\end{array}\right], \\
\varepsilon_{a} & =\left[\begin{array}{c}
w(t) \\
\dot{f}_{a}(t)
\end{array}\right] .
\end{aligned}
$$

Equations (17) and (18) can be rewritten as follows:

$$
\dot{\varphi}_{a}=A_{\mathrm{ma}} \varphi_{a}+B_{\mathrm{ma}} \varepsilon_{a},
$$

with

$$
\begin{aligned}
& A_{\mathrm{ma}}=\sum_{i=1}^{M} \mu_{i}(\xi(t)) A_{a i}, \\
& B_{\mathrm{ma}}=\sum_{i=1}^{M} \mu_{i}(\xi(t)) B_{a i},
\end{aligned}
$$

where

$$
\begin{aligned}
A_{a i} & =\left[\begin{array}{cc}
A_{i}-K_{i} C & E_{i} \\
-L_{i} C & 0
\end{array}\right], \\
B_{a i} & =\left[\begin{array}{ll}
-K_{i} D & 0 \\
-L_{i} D & I
\end{array}\right] .
\end{aligned}
$$

The matrix $I$ is the identity matrix with appropriate dimensions.

In order to analyse the convergence of the generalized estimation error $\varphi_{a}(t)$, the quadratic Lyapunov candidate function $V_{a}(t)=\varphi_{a}(t)^{T} P \varphi_{a}(t)$ is considered, where $P$ denotes a symmetric definite positive matrix.

The problem of robust state and fault estimation is reduced to find the gains $K_{i}$ and $L_{i}$ of the observer to ensure an asymptotic convergence of $\varphi_{a}(t)$ toward zero if $\varepsilon_{a}(t)=0$ and to ensure a bounded error in the case, where $\varepsilon_{a}(t) \neq 0$, i.e.,

$$
\begin{array}{ll}
\lim _{t \longrightarrow \infty} \varphi_{a}(t)=0, & \text { for } \varepsilon_{a}(t)=0, \\
\left\|\varphi_{a}(t)\right\|_{Q_{\varphi}} \leq \lambda\left\|\varepsilon_{a}(t)\right\|_{Q_{\varepsilon}} & \text { for } \varepsilon_{a}(t) \neq 0 \text { and } e(0)=0,
\end{array}
$$

where $\lambda>0$ is the attenuation level.

To satisfy constraints (23), it is sufficient to find a Lyapunov function $V_{a}(t)$ such that

$$
\dot{V}_{a}(t)+\varphi_{a}^{T} Q_{\varphi} \varphi_{a}-\lambda^{2} \varepsilon_{a}^{T} Q_{\varepsilon} \varepsilon_{a}<0,
$$

where $Q_{\varphi}$ and $Q_{\varepsilon}$ are two positive definite matrices.

In order to simplify the notations, the time index $(t)$ will be omitted henceforth.

Inequality (24) can also be written as follows:

$$
\psi_{a}^{T} \Omega_{a} \psi_{a}<0
$$

with

$$
\begin{aligned}
\psi_{a} & =\left[\begin{array}{c}
\varphi_{a} \\
\varepsilon_{a}
\end{array}\right], \\
\Omega_{a} & =\left[\begin{array}{cc}
A_{\mathrm{ma}}^{T} P+P A_{\mathrm{ma}}+Q_{\varphi} & P B_{\mathrm{ma}} \\
B_{\mathrm{ma}}^{T} P & -\lambda^{2} Q_{\varepsilon}
\end{array}\right] .
\end{aligned}
$$

Inequality (25) has a quadratic form, and it holds iff $\Omega_{a}<0$.

The matrices $A_{\mathrm{ma}}$ and $B_{\mathrm{ma}}$ can be written as

$$
\begin{aligned}
A_{\mathrm{ma}} & =\widetilde{A}_{\mathrm{ma}}-\widetilde{K}_{\mathrm{ma}} \widetilde{C}, \\
B_{\mathrm{ma}} & =\widetilde{I}-\widetilde{K}_{\mathrm{ma}} \widetilde{D},
\end{aligned}
$$

where

$$
\begin{aligned}
\widetilde{A}_{\mathrm{ma}} & =\sum_{i=1}^{M} \mu_{i}(\xi(t)) \widetilde{A}_{\mathrm{ma}}, \\
\widetilde{K}_{\mathrm{ma}} & =\sum_{i=1}^{M} \mu_{i}(\xi(t)) \widetilde{K}_{\mathrm{ma}},
\end{aligned}
$$

with

$$
\begin{aligned}
\widetilde{K}_{\text {mai }} & =\left[\begin{array}{c}
K_{i} \\
L_{i}
\end{array}\right], \\
\widetilde{A}_{\text {mai }} & =\left[\begin{array}{cc}
A_{i} & E_{i} \\
0 & 0
\end{array}\right], \\
\widetilde{I} & =\left[\begin{array}{ll}
0 & 0 \\
0 & I
\end{array}\right], \\
\widetilde{C} & =\left[\begin{array}{ll}
C & 0
\end{array}\right], \\
\widetilde{D} & =\left[\begin{array}{ll}
D & 0
\end{array}\right] .
\end{aligned}
$$

With the changes of variables $G_{\mathrm{ma}}=P \widetilde{K}_{\mathrm{ma}}$ and $\bar{\lambda}=\lambda^{2}$, the matrix $\Omega_{a}$ can be put as follows:

$$
\Omega_{a}=\left[\begin{array}{cc}
\vartheta_{a} & -G_{\mathrm{ma}} \widetilde{D}+P \widetilde{I} \\
\widetilde{I}^{T} P-\widetilde{D}^{T} G_{\mathrm{ma}}^{T} & -\bar{\lambda} Q_{\varepsilon}
\end{array}\right],
$$

where $\vartheta_{a}=P \widetilde{A}_{\mathrm{ma}}+\widetilde{A}_{\mathrm{ma}}^{T} P-G_{\mathrm{ma}} \widetilde{C}-\widetilde{C}^{T} G_{\mathrm{ma}}^{T}+Q_{\varphi}$.

As $\Omega_{a}=\sum_{i=1}^{M} \mu_{i}(\xi(t)) \Omega_{a i}$, the negativity of $\Omega$ is assured if, for $i=1, \ldots, M$,

$$
\Omega_{\mathrm{ai}}<0,
$$

with

$$
\Omega_{\mathrm{ai}}=\left[\begin{array}{cc}
\vartheta_{\mathrm{ai}} & -G_{\mathrm{ai}} \widetilde{D}+P \widetilde{I} \\
\widetilde{I}^{T} P-\widetilde{D}^{T} G_{\mathrm{ai}}^{T} & -\bar{\lambda} Q_{\varepsilon}
\end{array}\right],
$$

where $\vartheta_{\mathrm{ai}}=P \widetilde{A}_{\mathrm{mai}}+\widetilde{A}_{\mathrm{mai}}^{T} P-G_{\mathrm{ai}} \widetilde{C}-\widetilde{C}^{T} G_{\mathrm{ai}}^{T}+Q_{\varphi} \quad$ and $G_{\mathrm{ai}}=P \widetilde{K}_{\text {mai }}$.

The resolution of LMI (31) leads to the determination of the matrices $P$ and $G_{\text {ai }}$ and the scalar $\bar{\lambda}$. The gain matrices are then deduced by the equation $\widetilde{K}_{\text {mai }}=P^{-1} G_{\text {ai }}$.

The observer design is summarized by the following theorem.

Theorem 1. System (20) describing the time evolution of the state estimation error $\tilde{x}$ and the fault estimation error $\tilde{f}_{a}$ is stable and the $\mathscr{L}_{2}$-gain of the transfer from $\varepsilon_{a}(t)$ to $\varphi_{a}(t)$ is bounded if there exists a symmetric, positive definite matrix $P$, gain matrices $G_{a i}, i \in\{1, \ldots, M\}$, and a positive scalar $\bar{\lambda}$ such that the following LMI are verified: 


$$
\left[\begin{array}{cc}
\vartheta_{\mathrm{ai}} & -G_{\mathrm{ai}} \widetilde{D}+P \widetilde{I} \\
\widetilde{I}^{T} P-\widetilde{D}^{T} G_{\mathrm{ai}}^{T} & -\bar{\lambda} Q_{\varepsilon}
\end{array}\right]<0, \quad i \in\{1, \ldots, M\},
$$

where $\vartheta_{a i}=P \widetilde{A}_{m a i}+\widetilde{A}_{m a i}^{T} P-G_{a i} \widetilde{C}-\widetilde{C}^{T} G_{a i}^{T}+Q_{\varphi}$. The observer gains (proportional and integral gains) are computed using $\widetilde{K}_{m a i}=P^{-1} G_{a i}$ and the attenuation level is given by $\lambda=\sqrt{\lambda}$.

\section{Sensor Faults' Estimation}

The objective of this part is to adapt the proportional integral observer proposed in Section 3 to estimate sensor faults affecting the nonlinear system described by interval type 2 Takagi-Sugeno fuzzy model.

Let us consider the following interval type 2 Takagi-Sugeno system affected by sensor fault $f_{s}(t)$ and measurement noise $w(t)$ :

$$
\left\{\begin{array}{l}
\dot{x}(t)=\sum_{i=1}^{M} \mu_{i}(\xi(t))\left(A_{i} x(t)+B_{i} u(t)\right), \\
y(t)=C x(t)+F f_{s}(t)+D w(t),
\end{array}\right.
$$

where $x(t) \in \mathbb{R}^{n}$ is the system state, $y(t) \in \mathbb{R}^{m}$ is the measured output, $u(t) \in \mathbb{R}^{r}$ is the system input, $A_{i}, B_{i}$, and $C$ are known constant matrices with appropriate dimensions, $F$ and $D$ are, respectively, the fault and noise distribution matrices which are assumed to be known, the scalar $M$ is the number of local models, and $\mu_{i}(\xi(t))$ are the activation functions verifying equation (12).

Consider the state $z(t) \in \mathbb{R}^{p}[8,13]$ given by

$$
\dot{z}(t)=\sum_{i=1}^{M} \mu_{i}(\xi(t))\left(-\bar{A}_{i} z(t)+\bar{A}_{i} C x(t)+\bar{A}_{i} F f_{s}(t)\right),
$$

where $-\bar{A}_{i}, i \in\{1, \ldots, M\}$ are stables matrices.

Remark 1. The introduced new state $z(t)$ has the form of a particular filter for the output of the system; it was initially extended to the context of Takagi-Sugeno models in [8], and it was used in [13]. The main advantage of this new state is to conceive an augmented system where all the faults affecting the initial system (actuator and sensor faults) appear as unknown inputs which let possible to use an augmented proportional integral observer to estimate this unknown input considered as actuator faults. The use of this state is important because the classic proportional integral observer allows only estimating actuator faults which let the impossible to estimate the sensor fault based on the classical proportional observer.

The augmented state $\mathbf{x}(t)=\left[\begin{array}{lll}x^{T}(t) & z^{T}(t)\end{array}\right]^{T}$ is introduced. It is given by equation (36):

$$
\left\{\begin{array}{l}
\dot{\mathbf{x}}(t)=\sum_{i=1}^{M} \mu_{i}(\xi(t))\left(A_{g i} \mathbf{x}(t)+B_{g i} u(t)+E_{g i} f_{s}(t)\right), \\
\mathbf{y}(t)=C_{g} \mathbf{x}(t)+D_{g} w(t),
\end{array}\right.
$$

with

$$
\begin{aligned}
A_{g i} & =\left[\begin{array}{cc}
A_{i} & 0 \\
\bar{A}_{i} C & -\bar{A}_{i}
\end{array}\right], \\
B_{g i} & =\left[\begin{array}{c}
B_{i} \\
0
\end{array}\right], \\
E_{g i} & =\left[\begin{array}{c}
0 \\
\bar{A}_{i} F
\end{array}\right], \\
C_{g} & =\left[\begin{array}{ll}
C & 0 \\
0 & I
\end{array}\right], \\
D_{g} & =\left[\begin{array}{l}
D \\
0
\end{array}\right] .
\end{aligned}
$$

A proportional integral observer is able to estimate simultaneously the augmented state $\mathbf{x}(t)$ and the sensor fault $f_{s}(t)$ is chosen as follows:

$$
\left\{\begin{array}{l}
\dot{\hat{\mathbf{x}}}(t)=\sum_{i=1}^{M} \mu_{i}(\xi(t))\left(A_{g i} \widehat{\mathbf{x}}(t)+B_{g i} u(t)+E_{g i} \hat{f}_{s}(t)+K_{i} \widetilde{\mathbf{y}}(t)\right), \\
\hat{f}_{s}(t)=\sum_{i=1}^{M} \mu_{i}(\xi(t))\left(L_{i} \widetilde{\mathbf{y}}(t)\right), \\
\widehat{\mathbf{y}}(t)=C_{g} \widehat{\mathbf{x}}(t),
\end{array}\right.
$$

where $\widehat{\mathbf{x}}(t)$ is the estimated system state, $\widehat{f}_{s}(t)$ is the estimated sensor fault, $\hat{\mathbf{y}}(t)$ is the estimated output, $\widetilde{\mathbf{y}}(t)=\mathbf{y}(t)-\widehat{\mathbf{y}}(t), K_{i}$ are the local proportional observer gains, and $L_{i}$ are the local integral observer gains to be computed. It is assumed that $f_{s}(t)$ is bounded.

The augmented state estimation error $\widetilde{\mathbf{x}}(t)$ and the fault estimation error $\widetilde{f}_{s}(t)$ are defined as follows:

$$
\begin{gathered}
\widetilde{\mathbf{x}}(t)=\mathbf{x}(t)-\widehat{\mathbf{x}}(t), \\
\tilde{f}_{s}(t)=f_{s}(t)-\widehat{f}_{s}(t) .
\end{gathered}
$$

The dynamics of the augmented state reconstruction error is given by the computation of $\dot{\widetilde{\mathbf{x}}}(t)=\dot{\mathbf{x}}(t)-\dot{\widehat{\mathbf{x}}}(t)$ which is written as follows:

$$
\dot{\tilde{\mathbf{x}}}(t)=\sum_{i=1}^{M} \mu_{i}(\xi(t))\left(\left(A_{a i}-K_{i} C_{a}\right) \widetilde{\mathbf{x}}(t)+E_{a i} \tilde{f}_{s}(t)+K_{i} D_{a} w(t)\right) .
$$

The dynamic of the sensor fault estimation error can be computed as follows:

$$
\dot{\tilde{f}}_{s}(t)=\dot{f}_{s}(t)-\sum_{i=1}^{M} \mu_{i}(\xi(t))\left(L_{i} C_{a} \widetilde{\mathbf{x}}(t)-L_{i} D_{a} w(t)\right) .
$$

The following matrices are introduced: 


$$
\begin{aligned}
\varphi_{s} & =\left[\begin{array}{c}
\mathbf{x}(t) \\
\tilde{f}_{s}(t)
\end{array}\right], \\
\varepsilon_{s} & =\left[\begin{array}{c}
w(t) \\
\dot{f}_{s}(t)
\end{array}\right] .
\end{aligned}
$$

By omitting to denote the dependence with regard to the time $t$, equations (41) and (42) can be rewritten as follows:

$$
\dot{\varphi}_{s}=A_{\mathrm{ms}} \varphi_{s}+B_{\mathrm{ms}} \varepsilon_{s} .
$$

The matrices $A_{\mathrm{ms}}$ and $B_{\mathrm{ms}}$ have the following expressions:

$$
\begin{aligned}
A_{\mathrm{ms}} & =\sum_{i=1}^{M} \mu_{i}(\xi(t)) \widetilde{A_{s i}}, \\
B_{\mathrm{ms}} & =\sum_{i=1}^{M} \mu_{i}(\xi(t)) \widetilde{B_{s i}},
\end{aligned}
$$

where

$$
\begin{aligned}
& \widetilde{A_{s i}}=\left[\begin{array}{cc}
A_{a i}-K_{i} C_{a} & E_{a i} \\
-L_{i} C_{a} & 0
\end{array}\right], \\
& \widetilde{B_{s i}}=\left[\begin{array}{cc}
-K_{i} D_{a} & 0 \\
-L_{i} D_{a} & I
\end{array}\right] .
\end{aligned}
$$

Using the Lyapunov function $V_{s}(t)$ given by $V_{s}(t)=$ $\varphi_{s}(t)^{T} P \varphi_{s}(t)$ and following the same reasoning as for actuator faults' estimation, the convergence of state and fault estimation errors as well as the attenuation level is guaranteed if $\psi_{s}^{T} \Omega_{s} \psi_{s}<0$ with

$$
\begin{aligned}
\psi_{s} & =\left[\begin{array}{c}
\varphi_{s} \\
\varepsilon_{s}
\end{array}\right], \\
\Omega_{s} & =\left[\begin{array}{cc}
A_{\mathrm{mg}}^{T} P+\mathrm{PA}_{\mathrm{mg}}+Q_{\varphi} & \mathrm{PB}_{\mathrm{mg}} \\
B_{\mathrm{mg}}^{T} P & -\lambda^{2} Q_{\varepsilon}
\end{array}\right] .
\end{aligned}
$$

The matrices $A_{\mathrm{ms}}$ and $B_{\mathrm{ms}}$ are written as $A_{\mathrm{ms}}=\widetilde{A}_{\mathrm{ms}}-$ $\widetilde{K}_{\mathrm{ms}} \widetilde{C}_{g}$ and $B_{\mathrm{ms}}=\widetilde{I}-\widetilde{K}_{\mathrm{ms}} \widetilde{D}_{g}$ with

$$
\begin{gathered}
\widetilde{A}_{\mathrm{ms}}=\sum_{i=1}^{M} \mu_{i}(\xi(t)) \widetilde{A}_{\mathrm{msi}}, \\
\widetilde{K}_{\mathrm{ms}}=\sum_{i=1}^{M} \mu_{i}(\xi(t)) \widetilde{K}_{\mathrm{msi}},
\end{gathered}
$$

where

$$
\begin{aligned}
\widetilde{A}_{\mathrm{msi}} & =\left[\begin{array}{cc}
A_{\mathrm{gi}} & E_{\mathrm{gi}} \\
0 & 0
\end{array}\right], \\
\widetilde{K}_{\mathrm{msi}} & =\left[\begin{array}{c}
K_{i} \\
L_{i}
\end{array}\right], \\
\widetilde{I} & =\left[\begin{array}{ll}
0 & 0 \\
0 & I
\end{array}\right], \\
\widetilde{C}_{g} & =\left[\begin{array}{ll}
C_{g} & 0
\end{array}\right], \\
\widetilde{D}_{g} & =\left[\begin{array}{ll}
D_{g} & 0
\end{array}\right] .
\end{aligned}
$$

Using the changes of variables $G_{\mathrm{ms}}=P \widetilde{K}_{\mathrm{ms}}$ and $\bar{\lambda}=\lambda^{2}$ and choosing $Q_{\varphi}=Q_{\varepsilon}=I$, the matrix $\Omega_{s}$ can be put in the following form:

$$
\Omega_{s}=\left[\begin{array}{cc}
\vartheta_{s} & P \widetilde{I}-G_{\mathrm{ms}} \widetilde{D}_{a} \\
\widetilde{I}^{T} P-\widetilde{D}_{a}^{T} G_{\mathrm{ms}}^{T} & -\bar{\lambda} I
\end{array}\right],
$$

with $\vartheta_{s}=P \widetilde{A}_{\mathrm{ms}}+\widetilde{A}_{\mathrm{ms}}^{T} P-G_{\mathrm{ms}} \widetilde{C}_{a}-\widetilde{C}_{a}^{T} G_{\mathrm{ms}}^{T}+I$.

As $\Omega_{s}=\sum_{i=1}^{M} \mu_{i}(\xi(t)) \Omega_{\mathrm{si}}$, the negativity of $\Omega_{s}$ is assured if, for $i \in\{1, \ldots, M\}$,

$$
\Omega_{\mathrm{si}}<0,
$$

with

$$
\Omega_{\mathrm{si}}=\left[\begin{array}{cc}
\vartheta_{\mathrm{si}} & P \widetilde{I}-G_{\mathrm{si}} \widetilde{D}_{a} \\
\widetilde{I}^{T} P-\widetilde{D}_{a}^{T} G_{\mathrm{si}}^{T} & -\bar{\lambda} I
\end{array}\right],
$$

where $\vartheta_{\mathrm{si}}=P \widetilde{A}_{\mathrm{msi}}+\widetilde{A}_{\mathrm{msi}}^{T} P-G_{\mathrm{si}} \widetilde{C}_{a}-\widetilde{C}_{a}^{T} G_{\mathrm{si}}^{T}+I \quad$ and $G_{\mathrm{si}}=P \widetilde{K}_{\mathrm{msi}}$.

The resolution of LMI (51) leads to the determination of the matrices $P$ and $G_{\mathrm{si}}$ and the scalar $\bar{\lambda}$. The gain matrices are then deduced from the equation $\widetilde{K}_{\mathrm{msi}}=P^{-1} G_{\mathrm{si}}$. The observer design is summarized by the following theorem:

Theorem 2. System (44) describing the time evolution of the state estimation error $\tilde{x}$ and the fault estimation error $\tilde{f}$ is stable and the $\mathscr{L}_{2}$-gain of the transfer from $\varepsilon_{g}(t)$ to $\varphi_{g}(t)$ is bounded if there exists a symmetric, positive definite matrix $P$, gain matrices $G_{g i}, i \in\{1, \ldots, M\}$, and a positive scalar $m$ such that the following LMI is verified:

$$
\left[\begin{array}{cc}
\vartheta_{\mathrm{si}} & P \widetilde{I}-G_{\mathrm{gi}} \widetilde{D}_{a} \\
\widetilde{I}^{T} P-\widetilde{D}_{a}^{T} G_{\mathrm{gi}}^{T} & -\bar{\lambda} I
\end{array}\right]<0, \quad i \in\{1, \ldots, M\},
$$

with $\vartheta_{s i}=P \widetilde{A}_{m g i}+\widetilde{A}_{m g i}^{T} P-G_{g i} \widetilde{C}_{a}-\widetilde{C}_{a}^{T} G_{g i}^{T}+I$. The observer gains (proportional and integral gains) are computed using the equation $\widetilde{K}_{m g i}=P^{-1} G_{g i}$, and the attenuation level is given by $\lambda=\sqrt{\bar{\lambda}}$.

The main advantage of the proposed method is to estimate the sensor fault using a proportional integral observer. The use of the mathematical transformation (35) lets to estimate it since it appears as an actuator fault in the augmented system.

\section{Actuator and Sensor Faults' Estimation}

The objective of this part is to conceive a proportional integral observer which is able to estimate simultaneously actuator and sensor faults affecting the nonlinear system represented by interval type 2 Takagi-Sugeno model.

Let us consider the following nonlinear interval type 2 Takagi-Sugeno system affected by a sensor fault $f_{s}(t)$, actuator fault $f_{a}(t)$, and a measurement noise $w(t)$ :

$$
\left\{\begin{array}{l}
\dot{x}(t)=\sum_{i=1}^{M} \mu_{i}(\xi(t))\left(A_{i} x(t)+B_{i} u(t)+E_{i} f_{s}(t)\right), \\
y(t)=C x(t)+F f_{s}(t)+D w(t),
\end{array}\right.
$$


where $x(t) \in \mathbb{R}^{n}$ is the system state, $y(t) \in \mathbb{R}^{m}$ is the measured output, $u(t) \in \mathbb{R}^{r}$ is the system input, $A_{i}, B_{i}$, and $C$ are known constant matrices with appropriate dimensions, $E_{i}, F$, and $D$ are, respectively, the fault and noise distribution matrices which are assumed to be known, $M$ is the number of local models, and $\mu_{i}(\xi(t))$ are the activation functions verifying equation (12).

By considering the state $z(t) \in \mathbb{R}^{p}$ given in (35), the augmented state $\mathbf{x}(t)=\left[\begin{array}{ll}x^{T}(t) & z^{T}(t)\end{array}\right]^{T}$ is given by

$$
\left\{\begin{array}{l}
\dot{\mathbf{x}}(t)=\sum_{i=1}^{M} \mu_{i} \xi(t)\left(A_{\mathrm{gi}} \mathbf{x}(t)+B_{\mathrm{gi}} u(t)+W_{\mathrm{gi}} f(t)\right), \\
\mathbf{y}(t)=C_{g} \mathbf{x}(t)+D_{g} w(t),
\end{array}\right.
$$

with

$$
\begin{aligned}
A_{\mathrm{gi}} & =\left[\begin{array}{cc}
A_{i} & 0 \\
\bar{A}_{i} C & -\bar{A}_{i}
\end{array}\right], \\
B_{\mathrm{gi}} & =\left[\begin{array}{c}
B_{i} \\
0
\end{array}\right], \\
W_{\mathrm{gi}} & =\left[\begin{array}{cc}
E & 0 \\
0 & \bar{A}_{i} F
\end{array}\right], \\
C_{g} & =\left[\begin{array}{ll}
C & 0 \\
0 & I
\end{array}\right], \\
D_{g} & =\left[\begin{array}{c}
D \\
0
\end{array}\right], \\
f & =\left[\begin{array}{c}
f_{a} \\
f_{s}
\end{array}\right] .
\end{aligned}
$$

An adaptive structure of the proportional integral observer which is able to estimate simultaneously the system augmented state and the generalized fault $f(t)$ is chosen as follows:

$$
\left\{\begin{array}{l}
\dot{\hat{\mathbf{x}}}(t)=\sum_{i=1}^{M} \mu_{i}(\xi(t))\left(A_{\mathrm{gi}} \widehat{\mathbf{x}}(t)+B_{\mathrm{gi}} u(t)+W_{\mathrm{gi}} \widehat{f}(t)+K_{i} \widetilde{\mathbf{y}}\right), \\
\widehat{f}(t)=\sum_{i=1}^{M} \mu_{i}(\xi(t))\left(L_{i} \widetilde{\mathbf{y}}(t)\right), \\
\widehat{\mathbf{y}}(t)=C_{g} \widehat{\mathbf{x}}(t),
\end{array}\right.
$$

where $\widehat{\mathbf{x}}(t)$ is the estimated augmented system state, $\widehat{f}(t)$ is the estimated fault, $\widehat{\mathbf{y}}(t)$ is the estimated output, $\widetilde{\mathbf{y}}(t)=\mathbf{y}(t)-\widehat{\mathbf{y}}(t), K_{i}$ are the local proportional observer gains, and $L_{i}$ are the local integral observer gains to be computed. It is assumed that $f(t)$ is bounded.

Remark 2. The designed observer is called adaptive observer because it is based on the adaptive global model, and it lets the estimation of the actuator and the sensor fault after the application of the mathematical transformation. So, it has the same structure of a proportional integral observer, but this structure is adapted to the augmented system.
Let us define the augmented state estimation error $\widetilde{\mathbf{x}}(t)=$ $\mathbf{x}(t)-\widehat{\mathbf{x}}(t)$ and the fault estimation error $\widetilde{f}(t)=f(t)-\widehat{f}(t)$.

Their dynamics are given as follows:

$$
\dot{\widetilde{\mathbf{x}}}(t)=\sum_{i=1}^{M} \mu_{i}(\xi(t))\left(\left(A_{\mathrm{gi}}-K_{i} C_{g}\right) \widetilde{\mathbf{x}}(t)+E_{g i} \widetilde{f}(t)+K_{i} D_{g} w(t)\right),
$$

$\dot{\tilde{f}}(t)=\dot{f}(t)-\sum_{i=1}^{M} \mu_{i}(\xi(t))\left(L_{i} C_{g} \widetilde{\mathbf{x}}(t)-L_{i} D_{g} w(t)\right)$.

The following matrices are introduced:

$$
\begin{gathered}
\varphi_{f}=\left[\begin{array}{c}
\widetilde{\mathbf{x}}(t) \\
\tilde{f}(t)
\end{array}\right], \\
\varepsilon_{f}=\left[\begin{array}{c}
w(t) \\
\dot{f}(t)
\end{array}\right] .
\end{gathered}
$$

By omitting to denote the dependence with regard to the time $t$, equations (58) and (59) become

$$
\dot{\varphi}_{f}=A_{\mathrm{mf}} \varphi_{f}+B_{\mathrm{mf}} \varepsilon_{f}
$$

The matrices $A_{\mathrm{mf}}$ and $B_{\mathrm{mf}}$ have the expressions: $A_{\mathrm{mf}}=$ $\sum_{i=1}^{M} \mu_{i}(\xi(t)) \widetilde{A_{f i}}$ and $B_{\mathrm{mf}}=\sum_{i=1}^{m} \mu_{i}(\xi(t)) \widetilde{B_{f i}}$. with

$$
\begin{aligned}
& \widetilde{A_{\mathrm{fi}}}=\left[\begin{array}{cc}
A_{\mathrm{gi}}-K_{i} C_{g} & E_{\mathrm{gi}} \\
-L_{i} C_{g} & 0
\end{array}\right], \\
& \widetilde{B_{\mathrm{fi}}}=\left[\begin{array}{cc}
-K_{i} D_{g} & 0 \\
-L_{i} D_{g} & I
\end{array}\right] .
\end{aligned}
$$

The Lyapunov function $V_{f}(t)=\varphi_{f}(t)^{T} P \varphi_{f}(t)$ is considered. By following the same reasoning as for actuator faults' estimation, the convergence of state and fault estimation errors as well as attenuation level are guaranteed if $\psi_{f}^{T} \Omega_{f} \psi_{f}<0$ with

$$
\begin{aligned}
\psi_{f} & =\left[\begin{array}{c}
\varphi_{f} \\
\varepsilon_{f}
\end{array}\right], \\
\Omega_{f} & =\left[\begin{array}{cc}
A_{\mathrm{mf}}^{T} P+\mathrm{PA}_{\mathrm{mf}}+Q_{\varphi} & \mathrm{PB}_{\mathrm{mf}} \\
B_{\mathrm{mf}}^{T} P & -\lambda^{2} Q_{\varepsilon}
\end{array}\right] .
\end{aligned}
$$

The matrices $A_{\mathrm{mf}}$ and $B_{\mathrm{mf}}$ can be written as $A_{\mathrm{mf}}=\widetilde{A}_{\mathrm{mf}}-$ $\widetilde{K}_{\mathrm{mf}} \widetilde{C}_{g}$ and $B_{\mathrm{mf}}=\widetilde{I}-\widetilde{K}_{\mathrm{mf}} \widetilde{D}_{g}$ with

$$
\begin{aligned}
& \widetilde{A}_{\mathrm{mf}}=\sum_{i=1}^{M} \mu_{i}(\xi(t)) \widetilde{A}_{\mathrm{mfi}}, \\
& \widetilde{K}_{\mathrm{mf}}=\sum_{i=1}^{M} \mu_{i}(\xi(t)) \widetilde{K}_{\mathrm{mfi}},
\end{aligned}
$$

where 


$$
\begin{aligned}
\widetilde{A}_{\mathrm{mfi}} & =\left[\begin{array}{cc}
A_{\mathrm{gi}} & E_{\mathrm{gi}} \\
0 & 0
\end{array}\right], \\
\widetilde{K}_{\mathrm{mfi}} & =\left[\begin{array}{c}
K_{i} \\
L_{i}
\end{array}\right], \\
\widetilde{I} & =\left[\begin{array}{ll}
0 & 0 \\
0 & I
\end{array}\right], \\
\widetilde{C}_{g} & =\left[\begin{array}{ll}
C_{g} & 0
\end{array}\right], \\
\widetilde{D}_{g} & =\left[\begin{array}{ll}
D_{g} & 0
\end{array}\right] .
\end{aligned}
$$

Using the changes of variables $G_{\mathrm{mf}}=P \widetilde{K}_{\mathrm{mf}}$ and $\bar{\lambda}=\lambda^{2}$ and choosing $Q_{\varphi}=Q_{\varepsilon}=I$, the matrix $\Omega_{f}$ can be put in the following form:

$$
\Omega_{f}=\left[\begin{array}{cc}
\vartheta_{f} & P \widetilde{I}-G_{m f} \widetilde{D}_{a} \\
\widetilde{I}^{T} P-\widetilde{D}_{a}^{T} G_{m f}^{T} & -m I
\end{array}\right],
$$

with $\vartheta_{f}=P \widetilde{A}_{\mathrm{mf}}+\widetilde{A}_{\mathrm{mf}}^{T} P-G_{\mathrm{mf}} \widetilde{C}_{a}-\widetilde{C}_{a}^{T} G_{\mathrm{mf}}^{T}+I$.

As $\Omega_{f}=\sum_{i=1}^{M} \mu_{i}(\xi(t)) \Omega_{\mathrm{fi}}$, the negativity of $\Omega_{f}$ is assured if, for $i \in\{1, \ldots, M\}$,

$$
\Omega_{\mathrm{fi}}<0,
$$

with

$$
\Omega_{\mathrm{fi}}=\left[\begin{array}{cc}
\vartheta_{\mathrm{fi}} & P \widetilde{I}-G_{\mathrm{fi}} \widetilde{D}_{a} \\
\widetilde{I}^{T} P-\widetilde{D}_{a}^{T} G_{\mathrm{fi}}^{T} & -m I
\end{array}\right],
$$

where $\quad \vartheta_{\mathrm{fi}}=P \widetilde{A}_{\mathrm{mfi}}+\widetilde{A}_{\mathrm{mfi}}^{T} P-G_{\mathrm{fi}} \widetilde{\mathrm{C}}_{a}-\widetilde{C}_{a}^{T} G_{\mathrm{fi}}^{T}+I \quad$ and $G_{\mathrm{fi}}=P \widetilde{K}_{\mathrm{mfi}}$.

The resolution of LMI (67) leads to the determination of the matrices $P$ and $G_{\mathrm{fi}}$ and the scalar $m$. The gain matrices are then deduced using the equation: $\widetilde{K}_{\mathrm{mfi}}=P^{-1} G_{\mathrm{fi}}$.

The observer design is summarized by the following theorem.

Theorem 3. System (61) describing the time evolution of the state estimation error $\tilde{x}$ and the fault estimation error $\tilde{f}$ is stable and the $\mathscr{L}_{2}$-gain of the transfer from $\varepsilon_{f}(t)$ to $\varphi_{f}(t)$ is bounded if there exists a symmetric, positive definite matrix $P$, gain matrices $G_{f i}, i \in\{1, \ldots, M\}$, and a positive scalar $m$ such that the following LMI is verified:

$$
\left[\begin{array}{cc}
\vartheta_{\mathrm{fi}} & P \widetilde{I}-G_{\mathrm{fi}} \widetilde{D}_{a} \\
\widetilde{I}^{T} P-\widetilde{D}_{a}^{T} G_{\mathrm{fi}}^{T} & -m I
\end{array}\right]<0, \quad i \in\{1, \ldots, M\},
$$

where $\vartheta_{f i}=P \widetilde{A}_{m f i}+\widetilde{A}_{m f i}^{T} P-G_{f i} \widetilde{C}_{a}-\widetilde{C}_{a}^{T} G_{f i}^{T}+I$. The observer gains (proportional and integral gains) are computed using the equation $\widetilde{K}_{m f i}=P^{-1} G_{f i}$ and the attenuation level is given by $\lambda=\sqrt{\lambda}$.

The main advantage of this method is to estimate simultaneously the sensor and the actuator faults using a proportional integral observer. The use of the mathematical transformation 34 lets to obtain a generalized fault which combines the actuator and the sensor faults. This generalized fault appears as an actuator fault in the obtained augmented system. Its estimation leads to estimate the sensor and the actuator fault.

\section{Example of Simulation}

The objective of this section is to apply the proposed method to a hydraulic process made up of three tanks [37]. The system is supposed affected simultaneously by sensor and actuator faults. The three tanks $T_{1}, T_{2}$, and $T_{3}$ with identical sections $\rho$ are connected to each other by cylindrical pipes of identical sections $S_{n}$. The output valve is located at the output of $\operatorname{tank} T_{2}$; it ensures to empty the tank filled by the flow of pumps 1 and 2 with, respectively, flow rates $Q_{1}(t)$ and $a_{2}(t)$. Combinations of the three water levels are measured. The pipes of communication between the tanks are manually closed or open. The three levels $x_{1}, x_{2}$, and $x_{3}$ are governed by the constraint $x_{1}>x_{3}>x_{2}$; the process described by Figure 1 is modeled by equation (70). Taking into account the fundamental laws of conservation of the fluid, a nonlinear model is expressed by the following state equations [37]:

$$
\left\{\begin{array}{l}
\rho \frac{\mathrm{d} x_{1}(t)}{\mathrm{d} t}=-\alpha_{1} S_{n}\left(2 g\left(x_{1}(t)-x_{3}(t)\right)\right)^{1 / 2}+Q_{1}(t)+Q f_{1} f_{a}(t), \\
\rho \frac{\mathrm{d} x_{2}(t)}{\mathrm{d} t}=-\alpha_{3} S_{n}\left(2 g\left(x_{3}(t)-x_{2}(t)\right)\right)^{1 / 2}-\alpha_{2} S_{n}\left(2 g x_{2}(t)\right)^{1 / 2}+Q_{2}(t)+Q f_{2} f_{a}(t), \\
\rho \frac{\mathrm{d} x_{3}(t)}{\mathrm{d} t}=-\alpha_{1} S_{n}\left(2 g\left(x_{1}(t)-x_{3}(t)\right)\right)^{1 / 2}-\alpha_{3} S_{n}\left(2 g\left(x_{3}(t)-x_{2}(t)\right)\right)^{1 / 2}+Q f_{3} f_{a}(t),
\end{array}\right.
$$

where $\alpha_{1}, \alpha_{2}$, and $\alpha_{3}$ are constants, $f_{a}(t)$ is regarded as an unknown input, and $f_{s}(t)$ is a sensor fault affecting the process. $Q f / f i(t), i \in\{1, \ldots, 3\}$, denote the additional mass flows into the tanks caused by leaks and $g$ is the gravity constant.

The multiple model, with $\xi(t)=u(t)$, which approximates the nonlinear system (70), is given by the following state equation:

$$
\left\{\begin{array}{l}
\dot{x}(t)=\sum_{i=1}^{M} \mu_{i}(u(t))\left(A_{i} x(t)+B_{i} u(t)+E_{i} f_{s}(t)\right), \\
y(t)=C x(t)+F f_{s}(t)+D w(t) .
\end{array}\right.
$$

The matrices $A_{i}, B i$, and $d_{i}$ are calculated by linearizing the initial system (70) around four points chosen in the operation range of the system. Four local models have been selected in a heuristic way. That number guarantees a good 


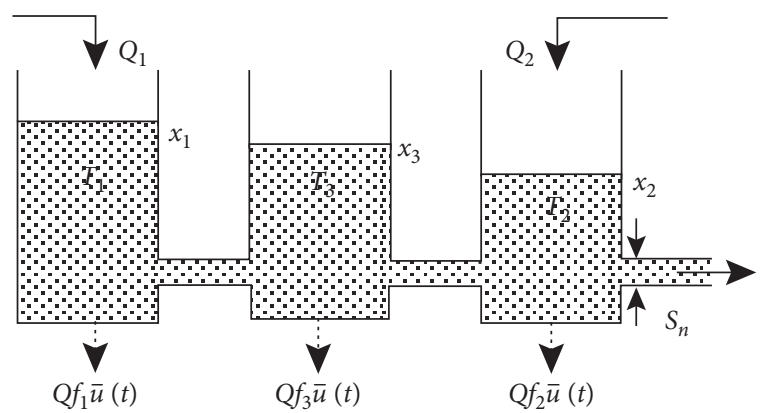

Figure 1: Three tanks' system.

approximation of the state of the real system by the multiple model [?]. The following numerical values were obtained:

$$
\begin{aligned}
& A_{1}=\left[\begin{array}{ccc}
-0.0109 & 0 & 0.0109 \\
0 & -0.0206 & 0.0106 \\
0.0109 & 0.0106 & -0.0215
\end{array}\right] \text {, } \\
& d_{1}=10^{-3} *\left[\begin{array}{c}
-2.86 \\
-0.38 \\
0.11
\end{array}\right] \text {, } \\
& A_{2}=\left[\begin{array}{ccc}
-0.0110 & 0 & 0.0110 \\
0 & -0.0205 & 0.0104 \\
0.0110 & 0.0104 & -0.0215
\end{array}\right] \text {, } \\
& d_{2}=10^{-3} *\left[\begin{array}{r}
-2.86 \\
-0.34 \\
0.038
\end{array}\right], \\
& A_{3}=\left[\begin{array}{ccc}
-0.0084 & 0 & 0.0084 \\
0 & -0.0206 & 0.0095 \\
0.0084 & 0.0095 & -0.0180
\end{array}\right] \text {, } \\
& d_{3}=10^{-3} *\left[\begin{array}{c}
-3.7 \\
-0.14 \\
0.69
\end{array}\right] \text {, } \\
& A_{4}=\left[\begin{array}{ccc}
-0.0085 & 0 & 0.0085 \\
0 & -0.0205 & 0.0095 \\
0.0085 & 0.0095 & -0.0180
\end{array}\right] \text {, } \\
& d_{4}=10^{-3} *\left[\begin{array}{c}
-3.67 \\
-0.18 \\
0.62
\end{array}\right] \\
& B_{i}=E_{i} \\
& D=\left[\begin{array}{ll}
0.1 & 0.5 \\
0.2 & 0.1 \\
0.1 & 0.3
\end{array}\right] \text {, } \\
& C=\left[\begin{array}{lll}
1 & 1 & 1 \\
1 & 0 & 0 \\
0 & 1 & 0
\end{array}\right] \text {. }
\end{aligned}
$$

The functions $Q f_{1}, Q f_{2}$, and $Q f_{3}$ are constant and the numerical application is made with

$$
\begin{aligned}
& Q f_{i}=10^{-4}, \forall i \in\{1, \ldots, 4\}, \text { and. } t \in[0, x,[ \\
& \alpha_{1}=0.78, \alpha_{2}=0.78, \text { and } \alpha_{3}=0.75 \\
& g=9.8, S_{n}=5 * 10^{-5} \text {, and } \rho=0.0154
\end{aligned}
$$

The type 2 activation functions are shown in Figure 2.

Figure 2 shows the evolution of activation functions $\mu_{i}(\xi(t))$ in time. For each activation function $\mu_{i}(\xi(t)), i \in 1, \ldots, 4$, the upper bound $\bar{\mu}_{i}(\xi(t))$ and the under bound $\mu_{i}(\xi(t))$ are represented. The evolution of the activation function in time is between these two bounds.

The actuator fault $f_{a}(t)=\left[\begin{array}{lll}f_{a 1}(t) & f_{a 2}(t)\end{array}\right]^{T}$ is defined as

$$
\begin{aligned}
& f_{a 1}= \begin{cases}0.4, & 100 \mathrm{~s}<t<700 \mathrm{~s}, \\
0, & \text { otherwise }\end{cases} \\
& f_{a 1}= \begin{cases}0.5, & 60 \mathrm{~s}<t<360 \mathrm{~s}, \\
0.8 * & \sin (0.2 \pi t), \quad 360 \mathrm{~s}<t<800 \mathrm{~s}, \\
0, & \text { otherwise. }\end{cases}
\end{aligned}
$$

The sensor fault $f_{s}(t)$ is $f_{s}(t)=\left[\begin{array}{lll}f_{s 1}(t) & f_{s 2}(t)\end{array}\right]^{T}$ with

$$
\begin{aligned}
& f_{s 1}= \begin{cases}0.7 * & \sin (0.4 \pi t), \quad 200 \mathrm{~s}<t<700 \mathrm{~s}, \\
0.6, & 700 \mathrm{~s}<t<1000 \mathrm{~s}, \\
0, & \text { otherwise, }\end{cases} \\
& f_{s 2}= \begin{cases}0.8 * & \sin (0.1 \pi t), \quad 350<t<550 \mathrm{~s}, \\
0.6, & 600 \mathrm{~s}<t<800 \mathrm{~s}, \\
0, & \text { otherwise. }\end{cases}
\end{aligned}
$$

Matrices $\bar{A}_{i}$ are chosen as $\bar{A}_{1}=5 * I, \bar{A}_{2}=10 * I$, $\bar{A}_{3}=15 * I$, and $\bar{A}_{4}=20 * I$.

Figure 3 visualizes the two actuator faults and their estimations. The actuator faults' error estimation is shown in Figure 4. In Figure 5, the two sensor faults and their estimations are represented, and the sensor faults' error estimation is shown in Figure 6. The state error estimation is visualized in Figure 7.

Figures 3 and 4 show that the proposed proportional observer allows estimating the actuator fault well even in the case of time-varying faults.

Figures 5 and 6 show that the proposed proportional observer allows estimating the sensor fault well even in the case of time-varying faults. Figure 7 shows also that this proposed observer allows estimating the system state well. The effect of the measurement noise is minimized using the $\mathscr{L}_{2}$ approach.

The obtained results show the effectiveness of the proposed proportional integral observer.

Figures 3 to 7 show that the proposed method gives a state and fault estimation with high performances. The proposed adaptive proportional observer lets to estimate the system state and the actuator and/or the sensor fault well. The proposed observer gives good results even in the case of time-varying faults. It is shown also that the proposed 


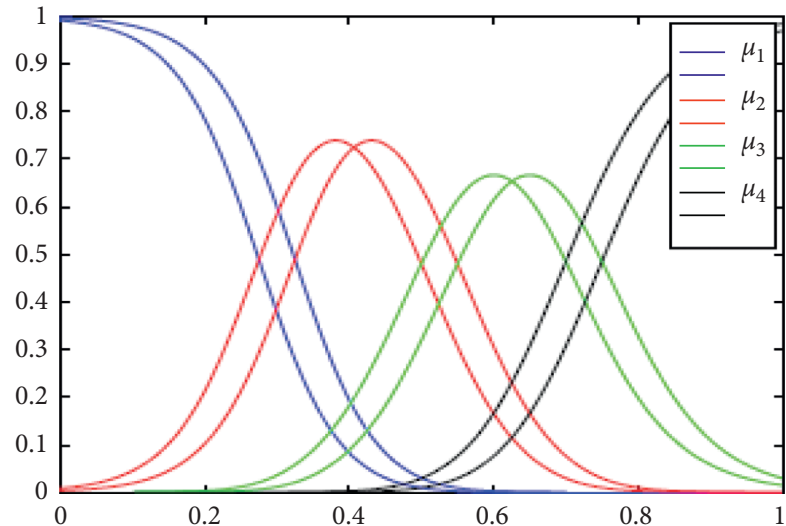

FIgURE 2: Actuator faults' estimation error.

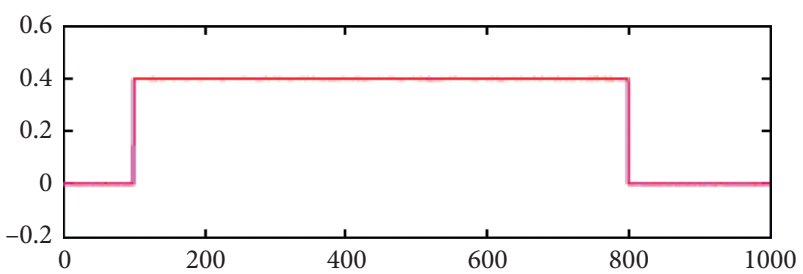

$-f_{a 1}$

- $f_{a 1}$ estimated

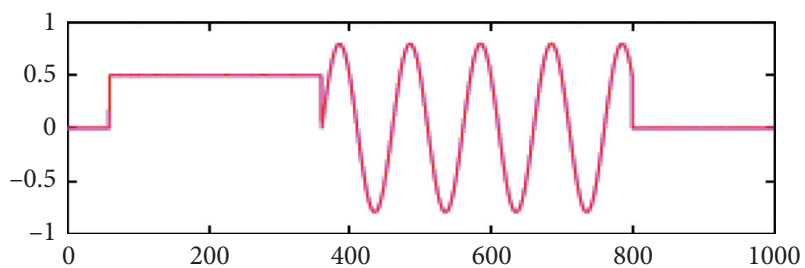

$f_{a 2}$

- $f_{a 2}$ estimated

FIGURE 3: Actuator faults and their estimation.
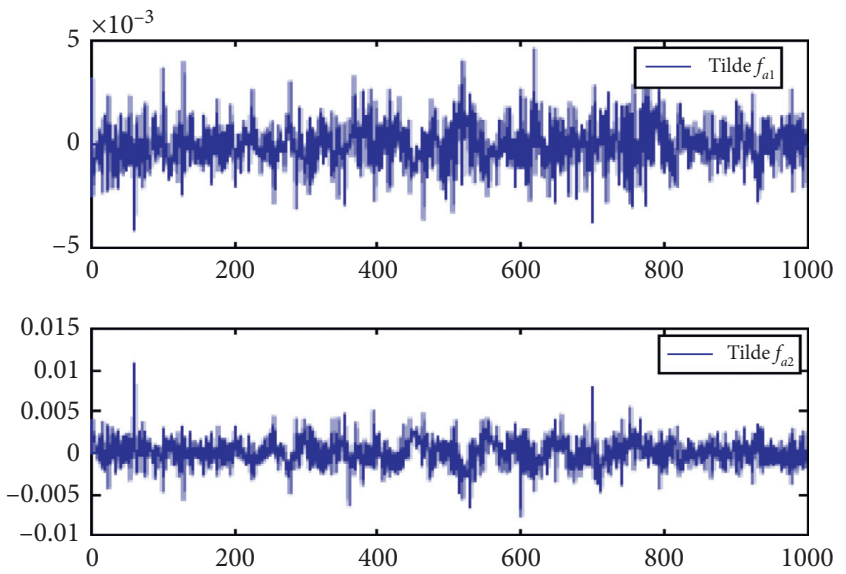

Figure 4: Actuator faults' estimation error.
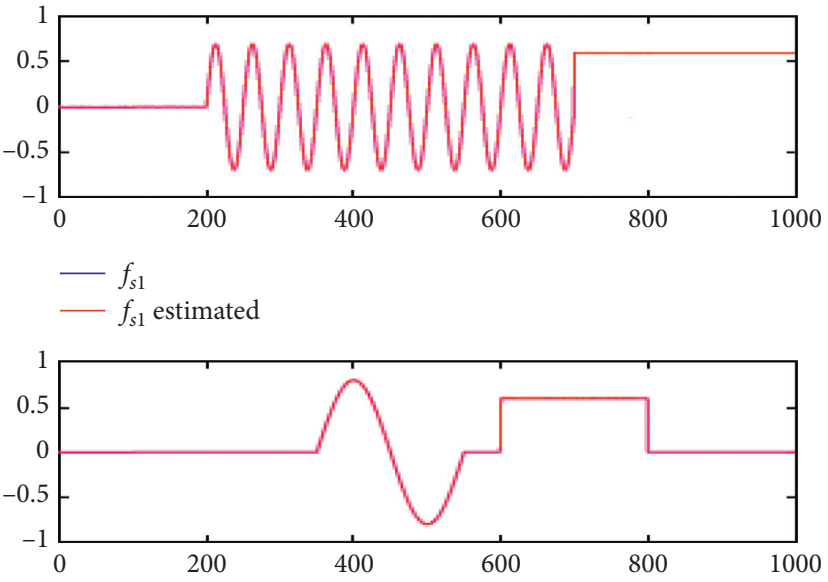

$-f_{s 2}$

- $f_{s 2}$ estimated

Figure 5: Sensor faults and their estimation.
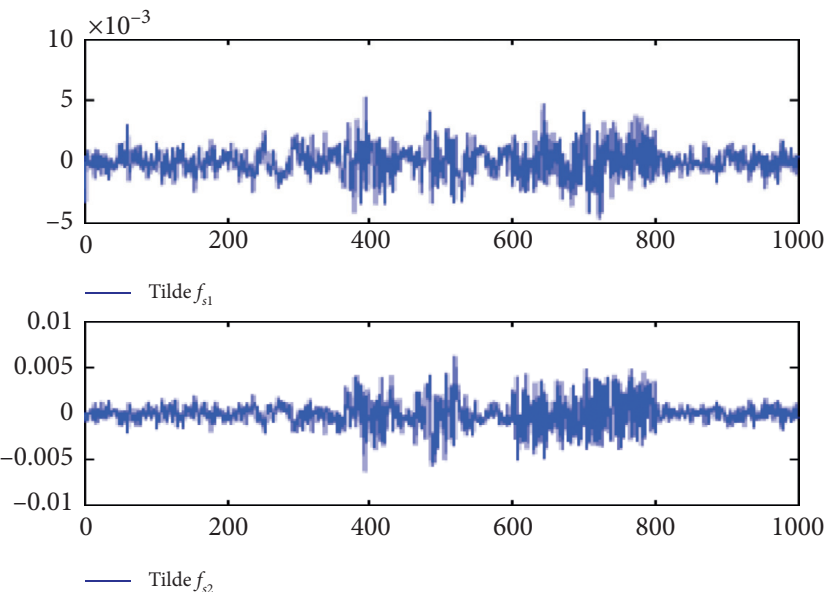

FIgURE 6: Sensor faults' estimation error.

observer is rapid and lets to estimate the system state and the fault in a very short time.

Simulations results present the robustness of the designed observer for state and fault estimation. Indeed, it is shown that the actuator fault and its estimation are nearly superposed. The same situation is obtained for sensor faults. The estimation error is less than $1 \%$ which is supposed acceptable estimation error for fault or state estimation. The analysis of simulation result lets to conclude that the conceived observer allows an acceptable state and fault estimation by its application to the three tanks system modeled by a type 2 interval Takagi-Sugeno model. The considered fault for the simulation is time varying which lets to obtain a general result without the assumption of null fault derivative. 

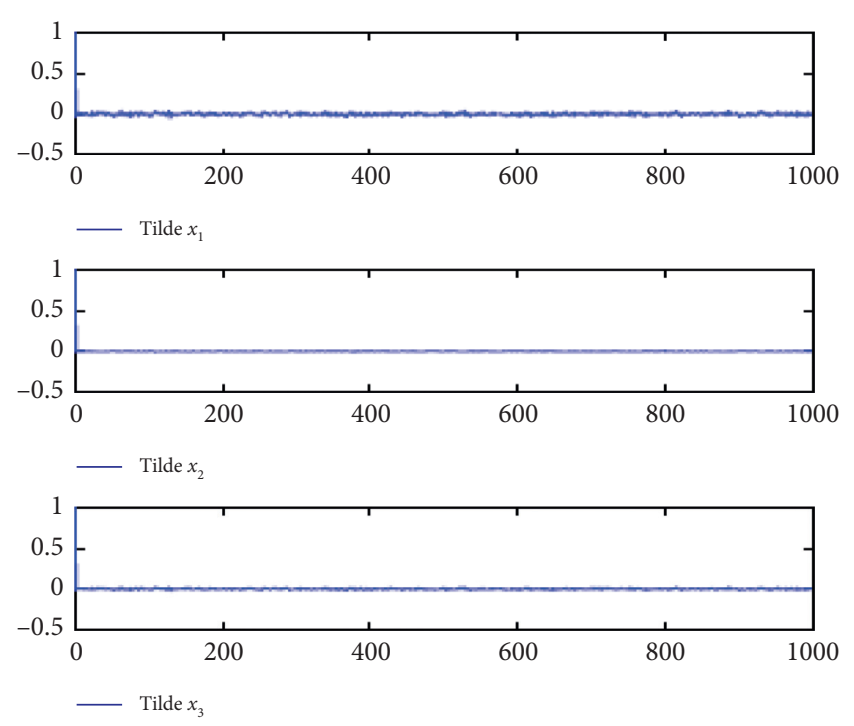

Figure 7: State estimation error.

Remark 3. The particularity of this work compared with others in the same context is that this work proposes an observer able to estimate actuator and sensor fault using a mathematical transformation which can be considered as a filter of the system output. Many works in this context suppose that the fault derivate is null, and this assumption is not considered in this work which makes it more general. It is shown that time-varying fault is well estimated.

\section{Conclusion}

This paper presents a method of a proportional integral observer design based on the principle of interval type 2 Takagi-Sugeno fuzzy systems. The proposed observer is able to estimate simultaneously the system state and the faults' affecting system. In this work, actuator faults are considered as unknown inputs. To estimate sensor faults, a mathematical transformation is used to conceive an augmented system in which the initial sensor fault appears as an unknown input. In this work, the system affected by actuator fault is considered firstly; then, the system affected by the sensor fault is treated. Moreover, the case, where the studied system is affected simultaneously by sensor and actuator faults, is considered. The computation of the global observer gains is reduced to the computation of the gains of the local observers.

This method allows estimating the faults well even in the case of time-varying faults. The noise effect on the state and fault estimation is also minimized using the $\mathscr{L}_{2}$ approach. The direct application of this observer could be the base for the design of a detection procedure and localization of faults. It is possible also to use the sensor and/or actuator fault estimation to conceive a fault tolerant control which can remain the evolution of the faulty system state to the state of the system where no faults are affecting it. In the same context of Takagi-Sugeno approach, more works will be developed taking into account more parameters such as system delays and fractional order systems. Other type of observers will be also developed, in particular, proportional multiple integral observers and observers with unknown input. These works will be extended in the second step to treat the problem of the design of fault tolerant control using the active approach.

\section{Data Availability}

No data were used to support this study.

\section{Conflicts of Interest}

The authors declare that there are no conflicts of interest regarding the publication of this article.

\section{References}

[1] N. Bouguila, W. Jamel, A. Khedher, and K. Ben Othman, "Multiple observer design for a non-linear Takagi-Sugeno system submitted to unknown inputs and outputs," IET Signal Processing, vol. 7, no. 8, pp. 635-645, 2013.

[2] C. Edwards, "A comparison of sliding mode and unknown input observers for fault reconstruction," in Proceedings of the 2004 43rd IEEE Conference on Decision and Control, vol. 5, pp. 5279-5284, Nassau, Bahamas, 2004.

[3] R. Sharma and M. Aldeen, Estimation of Unknown Disturbances in Nonlinear Systems, University of Bath, Bath, UK, 2004.

[4] I. Elleuch, A. Khedher, and K. B. Othman, "State and faults estimation based on proportional integral sliding mode observer for uncertain Takagi-Sugeno fuzzy systems and its application to a turbo-reactor," International Journal of Fuzzy Systems, vol. 19, no. 6, pp. 1768-1781, 2017.

[5] R. Orjuela, J. Ragot, D. Maquin, and B. Marx, "On the simultaneous state and unknown input estimation of complex systems via a multiple model strategy," IET Control Theory \& Applications, vol. 3, no. 7, pp. 877-890, 2009.

[6] K. Tanaka, T. Ikeda, and Y. Y. He, "Fuzzy regulators and fuzzy observers: relaxed stability conditions and LMI-based design," IEEE Transaction on Fuzzy Systems, vol. 6, no. 1, pp. 250-256, 1998.

[7] T. Takagi and M. Sugeno, "Fuzzy identification of systems and its applications to modeling and control," IEEE Transactions on Systems, Man, and Cybernetics, vol. SMC-15, no. 1, pp. 116-132, 1985.

[8] A. Khedher, K. Ben Othman, D. Maquin, and M. Benrejeb, "Sensor fault estimation for nonlinear systems described by Takagi-Sugeno models," Interntional Journal Transaction on System, Signal \& Devices, Issues on Systems, Signal \& Devices, vol. 6, no. 1, pp. 1-18, 2011.

[9] M. H. Asemani and V. J. Majd, "A robust $H_{\infty}$ non-PDC design scheme for singularly perturbed T-S fuzzy systems with immeasurable state variables," IEEE Transactions on Fuzzy Systems, vol. 23, no. 3, pp. 525-541, 2015.

[10] A. Kalhor, B. N. Araabi, and C. Lucas, "Evolving TakagiSugeno fuzzy model based on switching to neighboring models," Applied Soft Computing, vol. 13, no. 2, pp. 939-946, 2013.

[11] P. Radu-Emil, F. Horatiu-Ioan, R. Mircea-Bogdan, M. P. Emil, S. Preitl, and C. A. Dragos, "Online identification of evolving Takagi-Sugeno-Kang fuzzy models for crane systems," Applied Soft Computing, vol. 24, pp. 1155-1163, 2014. 
[12] D. Zhang, W. Cai, L. Xie, and Q.-G. Wang, "Nonfragile distributed filtering for T-S fuzzy systems in sensor networks," IEEE Transactions on Fuzzy Systems, vol. 23, no. 5, pp. 1883-1890, 2015.

[13] W. Jamel, A. Khedher, and K. B. Othman, "Observer design and active fault tolerant control for Takagi-Sugeno systems affected by sensors faults," International Journal of Modelling, Identification and Control, vol. 27, no. 1, pp. 22-30, 2017.

[14] K. Zhang, B. Jiang, and V. Coquempot, "Adaptive observerbased fast fault estimation," International Journal of Control, Automation, and Systems, vol. 6, no. 3, pp. 320-326, 2008.

[15] S. Fadali and S. Jafarzadeh, "Observer design for discrete type1 and type-2 TSK fuzzy systems," in Proceedings of the American Control Conference, Montréal, Canada, 2012.

[16] L. Hongyi, Y. Shen, P. Yingnan, and L. Hak-Keung, "Model reduction for interval type-2 Takagi-Sugeno fuzzy systems," Automatica, vol. 61, pp. 308-314, 2015.

[17] Q. Ren, M. Balazinski, and L. Baron, "Type-2 TSK fuzzy logic system and its type-1 counterpart," International Journal of Computer Applications, vol. 20, no. 6, 2011.

[18] H. Ying, "Interval type-2 Takagi-Sugeno fuzzy systems with linear rule consequent are universal approximators," in Proceedings of the 28th North American Fuzzy Information Processing Society Annual Conference (NAFIPS 2009), Cincinnati, OH, USA, June 2009.

[19] H. Jian, L. Xiuhua, G. Xianwen, and W. Xinjiang, "Intermediate observer-based robust distributed fault estimation for nonlinear multiagent systems with directed graphs," IEEE Transactions on Industrial Informatics, vol. 16, no. 12, pp. 7426-7436, 2020.

[20] L. Xiuhua, G. Xianwen, and H. Jian, "Distributed fault estimation for a class of nonlinear multiagent systems," IEEE Transactions on Systems, Man, and Cybernetics: Systems, vol. 50, no. 9, pp. 3382-3390, 2020.

[21] H. Kazumune, S. C. Michelle, and V. D. Dimos, "Distributed $\ell_{1}$-state-and-fault estimation for multiagent systems," IEEE Transactions on Control of Network Systems, vol. 7, pp. 699$710,2020$.

[22] L. Jianglin and P. Ron, "Asymptotic estimation of state, fault and perturbation for nonlinear systems and its fault-tolerant control application," International Journal of Control, Automation and Systems, vol. 19, no. 3, pp. 1175-1182, 2021.

[23] M. Ouzaz, A. El Assoudi, J. Soulami, and E. H. El Yaagoubi, "Simultaneous state and fault estimation for Takagi-Sugeno implicit models with Lipschitz constraints," An International Journal of Optimization and Control: Theories \& Applications (IJOCTA), vol. 11, no. 1, pp. 100-108, 2021.

[24] C. Martínez García, V. Puig, C. M. Astorga-Zaragoza, and G. L. Osorio-Gordillo, "Robust fault estimation based on interval Takagi-Sugeno unknown input observer," IFACPapersOnLine, vol. 51, no. 24, pp. 508-514, 2018.

[25] K. Ait Daraou, J. Soulami, A. El Assoudi, and E. H. El Yaagoubi, "State and fault observer design for discrete-time Takagi-Sugeno descriptor models," in Procedings of the 1st International Conference on Innovative Research in Applied Science, Engineering and Technology (IRASET), Meknes, Morocco, April 2020.

[26] R. Q. Emanoel, J. Chaves, F. O. Andre, A. Dantas, and A. L. Maitelli, "Unknown input observer-based actuator and sensor fault estimation technique for uncertain discrete time Takagi-Sugeno systems," International Journal of Control, Automation and Systems, vol. 19, no. 7, pp. 2444-2454, 2021.

[27] D. Zhao, L. Hak Keung, L. Yueyang, S. X. Ding, and L. Shuai, "A novel approach to state and unknown input estimation for
Takagi-Sugeno fuzzy models with applications to fault detection," IEEE Transactions on Circuits and Systems I: Regular Papers, vol. 67, no. 6, pp. 2053-2063, 2020.

[28] J. Qingxian, W. Lina, and L. Huayi, "Robust actuator fault reconstruction for Takagi-Sugeno fuzzy systems with timevarying delays via a synthesized learning and luenberger observer," International Journal of Control, Automation and Systems, vol. 19, no. 2, pp. 799-809, 2021.

[29] R. Hmidi, A. Ben Brahim, S. Dhahri, F. Ben Hmida, and A. Sellami, "Sliding mode fault-tolerant control for TakagiSugeno fuzzy systems with local nonlinear models: application to inverted pendulum and cart system," Transactions of the Institute of Measurement and Control, vol. 43, no. 4, 2021.

[30] A. Navarbaf and M. J. Khosrowjerdi, "Fault-tolerant controller design with fault estimation capability for a class of nonlinear systems using generalized Takagi-Sugeno fuzzy model," Transactions of the Institute of Measurement and Control, vol. 41, no. 15, 2019.

[31] M. Witczak, M. Pazera, N. Kukurowski, T. I. Bravo Cruz, and D. Theilliol, "Robust actuator and sensor fault estimation for Takagi-Sugeno fuzzy systems under ellipsoidal bounding," in Procedings of the IEEE International Conference on Fuzzy Systems (FUZZ-IEEE), Glasgow, UK, July 2020.

[32] W.-J. Chang, C.-C. Ku, and P.-H. Huang, "Robust fuzzy control for uncertain stochastic time-delay Takagi-Sugeno fuzzy models for achieving passivity," Fuzzy Sets and Systems, vol. 161, no. 15, pp. 2012-2032, 2010.

[33] J. Dong, G.-H. Yang, and H. Zhang, "Stability analysis of T-S fuzzy control systems by using set theory," IEEE Transactions on Fuzzy Systems, vol. 23, no. 4, pp. 827-841, 2015.

[34] M. A. Basil, J. Agustín, and G. L. Ramón, "Fuzzy optimal control using generalized Takagi-Sugeno model for multivariable nonlinear systems," Applied Soft Computing, vol. 30, pp. 205-213, 2015.

[35] N. Kumaresan and K. Ratnavelu, "Optimal control for stochastic linear quadratic singular neuro Takagi-Sugeno fuzzy system with singular cost using genetic programming," $A p$ plied Soft Computing, vol. 24, pp. 1136-1144, 2014.

[36] H. Moodi and M. Farrokhi, "Robust observer-based controller design for Takagi-Sugeno systems with nonlinear consequent parts," Fuzzy Sets and Systems, vol. 273, pp. 141-154, 2015.

[37] A. Akhenak, M. Chadli, J. Ragot, and D. Maquin, "State estimation via multiple observer with unknown input application to the three tank system," in Proceedings of the 5th IFAC Symposium on Fault Detection Supervision and Safety for Technical Processes, pp. 245-251, Washington, DC, USA, June 2003. 